\title{
sciendo
}

\section{The Effects of Compression Garments on Stability and Lower Limb Kinematics During a Forward Lunge}

\author{
by \\ Ioannis Angelakos ${ }^{1}$, Chris Mills ${ }^{1}$, Joseph O'Halloran ${ }^{1}$
}

\begin{abstract}
Compression garments have been used to minimise injury risk, through improvements in stability and joint positioning; yet, it is unclear whether there is an optimal length or tightness of these garments that may maximise observed benefits. This study measured the effect of three different garment types, at two different tightness levels, on lower extremity stability and alignment during a forward lunge movement. Sixteen healthy adults ( 7 female, 9 male; 24.3 \pm 2.9 years) were recruited as participants. Stability of the lead foot, as well as lower body joint kinematics, were recorded using an Oqus 12-camera system, surrounding participants as they executed three forward lunges onto a Matscan pressure mat under seven compression conditions (Control, Light/Heavy Calf, Light/Heavy Socks, Light/Heavy Leggings). Mean minimum time-to-boundary ( $m m T t B$ ) (derived from centre of pressure measures) and frontal plane kinematics (lateral pelvic tilt, knee valgus, ankle inversion/eversion) were used to assess the effect of garment tightness and length on lunge stability and joint alignment, respectively. A significant effect of tightness on mmTtB was observed $\left(F(1,105)=8.192 ; p=.005, \eta^{2}=.072\right)$, with Heavy garments eliciting longer mmTtB compared to their corresponding Light $(-.18 \pm .06 \mathrm{~s} ; p=.015)$ or Control $(-.28 \pm .09 \mathrm{~s} ; p=.007)$ conditions. No significant effects of garment tightness or length on lower body kinematics were evident. The results of this study suggest stability during a forward lunge is improved through the use of tight-fitted compression garments.
\end{abstract}

Key words: time-to-boundary, proprioception, injury, joint alignment.

\section{Introduction}

Single-limb joint control and stability are the key components to the functional status of the lower extremity (Paterno et al., 2004). Sub-optimal positions or instability during movement can cause unnatural loading of the musculoskeletal support structure. This often results in pronounced joint misalignment and injurious kinematics (Cook, 2009). The execution of a single-leg, forward lunge has been proposed as an effective screening tool for such injuries (Kritz et al., 2009). The lunge is part of most deceleration, single-leg jump or side-cutting motions (Cook, 2009), present in many sports such as basketball or rugby. During execution of an incorrect lunge, the hip, knee and ankle joints are misaligned; this increases the potential of injury occurrence (Kritz et al., 2009). Ideal lunge kinematics should demonstrate no lateral hip tilt (Powers, 2010), low knee valgus/varus (Boyle,
2012) and minimal ankle inversion/eversion (Hertel, 2002); thus the body stays in equilibrium, as the centre of mass remains within support base limits.

This maintenance of balance is paramount to injury prevention, as research has shown that centre of pressure $(\mathrm{CoP})$ measures during single leg standing can identify many neuromuscular deficits; CoP measures have been associated to anterior cruciate ligament (ACL) injuries (Paterno et al., 2010), ankle sprains (Hertel et al., 2001) and even injurious fall occurrence (Mak and $\mathrm{Ng}$, 2003). However, the use of simple $\mathrm{CoP}$ excursion and velocity measures for detecting instability has its limitations. Holme et al. (1999) revealed no significant differences between groups of injured and non-injured participants in either active or passive ankle joint-position sense in research using classic CoP measures. Also, the majority of

1 - School of Sport, Health and Exercise Sciences, University of Portsmouth, Portsmouth, UK.

Authors submitted their contribution to the article to the editorial board.

Accepted for printing in the Journal of Human Kinetics vol. 71/2020 in January 2020. 
research which supports the use of simple CoP excursion or velocity has focused only on injured populations (Hertel et al., 2001; Paterno et al., 2010); furthermore, little to no research has investigated stability during movement. A relatively novel method of assessing postural control involves using measures related to time-toboundary (TtB) of the CoP. Research by Hertel et al. (2006) has supported the use of TtB as a singleleg stance assessment tool, further encouraged by the results of research by Hertel and OlmstedKramer (2007); the latter observed that only TtB, and not traditional CoP measures, was able to detect motor deficiencies related to recurrent ankle sprains. $\mathrm{TtB}$ is measured by calculating excursions of the CoP from the support base limits of the foot being observed (Slobounov et al., 1997; van Wegen et al., 2002). Within this area of research, ideal lunge execution would also display larger $\mathrm{TtB}$ values; i.e. slower and smaller $\mathrm{CoP}$ excursions due to tighter neuromuscular control (van Emmerik and van Wegen, 2002).

Ergogenic compression garments have been utilized in attempts to sustain or improve joint kinematics (Lee et al., 2016; Mills et al., 2015; Varela-Sanz et al., 2011) and/or limb stability and balance control (Kuster et al., 1999; Michael et al., 2014; Pearce et al., 2009). While earlier research supported that any motor control/balance improvements were due to physiological effects of compression on venous flow (Sigel et al., 1975) and/or unnecessary motion reduction (Blair et al., 1995), subsequent research has challenged these explanations (Harris, 1996). It is now theorized that the movement benefits of compression clothing are most likely due to neurological effects (Pearce et al., 2009); more specifically by increased stimuli from sensory and motor systems (Riemann and Lephart, 2002). This information is usually defined with the umbrella term proprioception. Proprioception can mediate joint function and sensation, ensuring that the musculoskeletal system is in balance to overcome any overload on muscles and joints (Kuster et al., 1999). It has also been shown to inform postural control and instigate balance correction movements (Inglis et al., 1994). Lower extremity proprioception benefits have been observed in multiple studies investigating the effect of braces, taping or bandages on movements involving the knees and ankles; these benefits have been attributed to the increased stimuli from the skin and superficial muscles (Prymka et al., 1998) rather than just due to mechanical support. As such, it is understood that increased proprioceptor information received from compression garments is what results in any observed stability and kinematic benefits.

To date, there is no consensus on how specific garment attributes may influence these effects. Neuromuscular improvements have been observed in participants wearing items such as knee sleeves (Kuster et al., 1999), stockings (VarelaSanz et al., 2011), full length compression tights (Michael et al., 2014), and even full-body lycra garments (Rennie et al., 2000). These many clothing types can cover various combinations of joints and lower-body segments, which makes studying discrete effects challenging. Recently, Lee et al. (2016) investigated single-leg landing in participants wearing compression pants with differential pressurisation; the researchers observed that even small changes in the level, locus or method of pressurisation drastically changed kinematic profiles and stability measures. Aside from this, there is limited research on how garment specifics may relate to kinematic or stability measures and/or potentially affect them; does greater cutaneous stimulation (whether by increased length, tightness, or both) yield better results, or is there an ideal locus or intensity of compression that provides the most benefits movement kinematics or stability? As proprioceptive input from the legs informs the motor system (Riemann and Lephart, 2002), it would seem logical that between two garments of similar length, the tighter garment would provide more neuromuscular feedback (due to the enhanced compression on the joints and muscles), resulting in improved stability and joint control. Within this framework, a larger total area of cutaneous stimulation (i.e. a longer garment covering more joints) should also prove more beneficial to balance and movement quality. To date, only Dascombe and colleagues (2011) attempted to examine how different garment sizes (and tightness levels) of the same garment affected runners' performance, but focused only on performance and physiological data.

The aim of this study was to examine the effects of compression garments of varying lengths and tightness levels on stability and lower body joint alignment during a forward lunge. The first 
hypothesis states that there will be a significant decrease in mean minimum $\mathrm{TtB}$ (mmTtB) under the longer and tighter garment conditions. The second hypothesis states that there will be a significant decrease in lower-body kinematic deviations under the longer and tighter garment conditions.

\section{Methods}

\section{Participants}

Following institutional ethical approval, participants ( $\mathrm{n}=16 ; 7$ female, 9 male) with no current or recent (within the last 6 months of trial commencement) musculoskeletal injury or pathology that could influence movement or sensation of the lower body were recruited via email. Exclusion criteria included any surgeries of the trunk and lower body, osteoarthritis, knee joint effusion or current muscular/ligamentous injury. Participants were only included if they were not experiencing any pain, illness or voiced any complaints of pain during lunging. Written and informed consent was received from participants prior to the beginning of trials. Participants were all under 30 years of age $(24.3 \pm 2.9$ years $) ; 4$ identified as left leg dominant, 12 as right leg dominant. All participants were experienced in the performance of the lunge exercise. Lunge distance (from the starting position to the centre of the pressure mat) was standardised to leg length (83.6 $\pm 6.9 \mathrm{~cm}$ ), as measured from the greater trochanter to the floor while barefoot (Mills et al., 2015).

\section{Design and Procedures}

Prior to testing, participants conducted a brief warm-up; this consisted of 5 minutes of lowintensity treadmill running at a self-selected pace, followed by dynamic lower extremity stretching. Participants then removed any footwear and socks.

Participants were assigned to a series of 7 conditions (6 compression conditions, 1 control), in randomized order. The three garment types tested were: Leggings (full-length compression, covering the legs from the pelvis to above the ankle), Socks (covering the lower leg under the knee, including the ankle and foot), and Calf Sleeves (above the ankle, below the knee). Each garment type was administered at two compression levels: Heavy and Light. This was achieved by the participants wearing two garments of the same type for the Heavy conditions (e.g. two socks on each leg), compared to only one for the Light conditions. All compression clothing was available at 4 sizes (extra-large, large, medium, and small), sized according to guidelines of the manufacturers; this was done so as to accommodate all body types. The garments used were Nike® Pro Cool Senior Compression Tights TM, SLS3®Allrounder Compression Socks ${ }^{\mathrm{TM}}$ and SKINS® Essentials Compression MX Calf Tights ${ }^{\mathrm{TM}}$.

After being assigned to their conditions, participants wore the appropriate garment; maximum foot width was measured with a tape measure; 5 retro-reflective markers were then positioned onto predetermined anatomical landmarks on the hips and dominant leg using hypoallergenic tape (Figure 1). One was attached onto each of the left and right anterior superior iliac spines (ASIS), another at the centre of the patella, with the two ankle markers placed vertically on the posterior surface of the foot, onto the calcaneal tubercle and the other $1 \mathrm{~cm}$ below the axis of subtalar movement (middle of the Achilles tendon). While marker positioning was based on previous investigations of lower limb biomechanics within a global coordinate system (Ferber and Benson, 2011; Floyd, 2012; Mills et al., 2015), only the relevant landmarks to this study's variables were chosen. All trials were performed without footwear. 12 Oqus Cameras (QualisysOqus 300/310, Sweden) with a sampling frequency of $100 \mathrm{~Hz}$ were set up, surrounding the lunge area in order to record the markers' positional coordinates. Calibration of activity volume was achieved using a calibration wand and L-shaped frame (Qualisys, Sweden). Tape was positioned on the floor along the $\mathrm{x}$ axis of the movement (identifying the anterioposterior plane; tape marking also used to signify the starting position) and the global coordinate system was set to identify $\mathrm{x}$ as the anterioposterior line, $\mathrm{y}$ as mediolateral and $\mathrm{z}$ as vertical. A MatScan pressure mat (ClassicMat, Tekscan, USA) sampling at a frequency of $40 \mathrm{~Hz}$ was used to gather centre of force data between the points of first and final contact of the foot with the ground, which was necessary for subsequent calculation of $\mathrm{TtB}$.

Trials involved execution of 3 bodyweight, forward lunges, landing onto the foot of the dominant leg; the movement was along the $\mathrm{x}$ axis onto a pressure mat until the hips were horizontally aligned to the lead knee, before returning back to the starting position. Participants 
were instructed to keep their torso upright at all times, with no leaning of the trunk or head; both feet should always be pointing forwards, with the hands laced behind the head and the front knee aligned over the lead ankle (Cook, 2009; Kritz et al., 2009). Any excessive imbalance, fall or sliding/slipping of the foot would be noted (as a failed trial), and the lunge repeated. Instructions were also given to aim for foot contact at the centre of the pressure mat. After execution of 3 successful trials, participants were given 3 minutes to change into the garments corresponding to the next condition and repeated this process, until all 21 lunge trials were completed.

Qualisys Track Manager (2.6, Qualisys Track Manager, Sweden) was used to identify markers. Any gaps in the trajectories were linearly interpolated, and a second order low pass Butterworth filter with a cut off frequency of $10 \mathrm{~Hz}$ was used for data filtering. The variables of interest - maximal lateral pelvic tilt, maximal knee valgus and maximal ankle eversion/inversion during each lunge - were then calculated using the processed data; all variables were measured in degrees $\left(^{\circ}\right)$. The maximum lateral pelvic tilt angle was computed between the contralateral and ipsilateral anterior superior iliac spine (ASIS) relative to the $\mathrm{XY}$ plane. The maximal valgus of the knee was calculated between the ipsilateral ASIS and patella relative to the XZ plane. Maximal eversion (+) and inversion (-) of the ankle were computed using the angle produced by the upper and lower calcaneus (ipsilateral) in the XZ plane. Only values recorded between the initiation of and the cessation of lead foot contact (at the end of the lunge) were reported. Data were averaged over 3 lunges.

Mediolateral centre of pressure $(\mathrm{CoP})$ data acquired from the pressure mat (MatScan software) were used to compute $\mathrm{TtB}$ information. In order to calculate $\mathrm{TtB}$, stability boundaries (Figure 2) are generally defined as a rectangle derived from the maximum foot width and length (Hertel el al., 2006). However, due to the nature of this study, only the mediolateral edges of the rectangle were used as boundaries. As the CoP can never actually reach or surpass these given boundaries during a successful lunge (as that would mean a fall/loss of balance occurred), timeto-boundary cannot be measured directly. However, this can be achieved indirectly by measuring $\mathrm{TtB}$ as it approaches a point of 'turning around' (i.e a moment when velocity goes to zero). By using the instantaneous mediolateral $\mathrm{CoP}$ position in relation to the origin, and the fixed time difference between frames, instantaneous CoP velocity values were calculated. Subsequently, instantaneous distance in relation to mediolateral stability boundaries and mediolateral velocity of the CoP were used to produce a $\mathrm{TtB}$ time series (Figure 3) of the CoP (van Wegen et al., 2001). Values of minimum time to contact were then averaged, resulting in a single mean of $\mathrm{TtB}$ minima (mmTtB) per lunge. Standard deviation (SD) of mmTtB was also calculated, as a reduction in minimum $\mathrm{TtB}$ variability is thought to be representative of a more constrained sensorimotor system (McKeon and Hertel, 2008). All data were averaged between the 3 trials under each condition.

\section{Statistical Analyses}

$\mathrm{TtB}$ measures were statistically analyzed using SPSS statistical software version 24 (IBM Corp, Armonk, NY, USA), as were foot, leg and hip kinematics. All data were checked for normality (Shapiro-Wilk test). Three separate $3 \times 4$ two-way ANOVAs were used to determine if any significant effects of tightness level and/or garment type on maximal lateral pelvic tilt, maximal knee valgus, maximal ankle eversion/inversion and mmTtB occurred. Wherever significant interactions were found by an ANOVA, post-hoc testing, with a Bonferroni correction applied, was used to identify any significant differences between specific conditions. The alpha level was set at $p \leq 0.05$ for all tests.

\section{Results}

\section{Maximal lateral pelvic tilt}

The greatest lateral pelvic tilt angle occurred under the Control condition $\left(9.1 \pm 3.4^{\circ}\right)$, whereas the least was observed under the Heavy Leggings condition $\left(7.4 \pm 3.4^{\circ}\right)$; however there were no significant effects of the compression level $\left(\mathrm{F}_{(1,104)}=.298 ; p=.586, \eta^{2}=.003\right)$ or the garment type $\left(\mathrm{F}_{(2,105)}=1.18 ; p=.311, \eta^{2}=.022\right)$ on lateral pelvic tilt values (Table 1). Additionally, there was no significant interaction effect between the compression level and the garment type $\left(\mathrm{F}_{(2,105)}=\right.$ $\left..072 ; p=.93, \eta^{2}=.001\right)$.

Maximal knee valgus

The greatest knee valgus angle was observed under the Heavy Leggings condition 
$\left(16 \pm 3.5^{\circ}\right)$, with the smallest angle measured under the Heavy Calf condition $\left(13.5 \pm 3.9^{\circ}\right)$; however, there were no significant effects of the compression level $\left(\mathrm{F}_{(1,105)}=.042 ; p=.838, \eta^{2}<.001\right)$ or the garment type $\left(\mathrm{F}_{(2,105)}=2.164 ; p=.12, \eta^{2}=.04\right)$ on knee valgus angle values. Additionally, there was no statistically significant interaction effect between the compression level and the garment type $\left(\mathrm{F}_{(2,105)}\right.$ $\left.=.522 ; p=.595, \eta^{2}=.01\right)$.

Maximal ankle inversion / eversion

The greatest ankle inversion angle was measured under the Light Leggings condition (7.3 $\pm 4.4^{\circ}$ ), whereas the smallest occurred under the Control condition $\left(3.5 \pm 2.3^{\circ}\right)$. No significant effects of the compression level $\left(\mathrm{F}_{(1,105)}=.076 ; p=.783, \eta^{2}=\right.$ $.001)$ or the garment type $\left(\mathrm{F}_{(2,105)}=1.348 ; p=.264, \eta^{2}\right.$ $=.025)$ on ankle inversion were observed. Also, there were no significant interaction effects $\left(\mathrm{F}_{(2,105)}\right.$ $=1.986 ; p=.142, \eta^{2}=.036$ ) between the compression level and the garment type. The largest ankle eversion was evident under the Light Socks condition $\left(6.1 \pm 5.9^{\circ}\right)$ with the least measured under the Light Leggings condition $\left(1.9 \pm 5.1^{\circ}\right)$. No significant effect of the garment compression level $\left(\mathrm{F}_{(1,105)}=.014 ; p=.906, \eta^{2}<.001\right)$ or the garment type $\left(\mathrm{F}_{(2,105)}=1.829 ; p=.166, \eta^{2}=.034\right)$ on eversion was revealed; furthermore, no significant interaction effect was observed $\left(\mathrm{F}_{(2,105)}=.946 ; p=.392, \eta^{2}=.018\right)$ between the compression level and the garment type.

Mean minimum TtB measures

Statistical analysis revealed that the shortest mmTtB occurred under the Control condition ( 0.51 $\pm 0.19 \mathrm{~s})$, with the longest $\mathrm{mmTtB}$ being observed under the Heavy Socks condition $(0.87 \pm 0.57 \mathrm{~s})$. No significant effects of the garment type on $\mathrm{mmTtB}$ were found $\left(\mathrm{F}_{(2,105)}=.382 ; p=.684, \eta^{2}=.007\right)$; there was also no significant interaction effect $\left(\mathrm{F}_{(2,105)}=\right.$ .799; $\left.p=.452, \eta^{2}=.015\right)$. However, results revealed a significant effect of the compression level on $\operatorname{mmTtB}\left(\mathrm{F}_{(1,105)}=8.192 ; p=.005, \eta^{2}=.072\right)$.

Figure 1

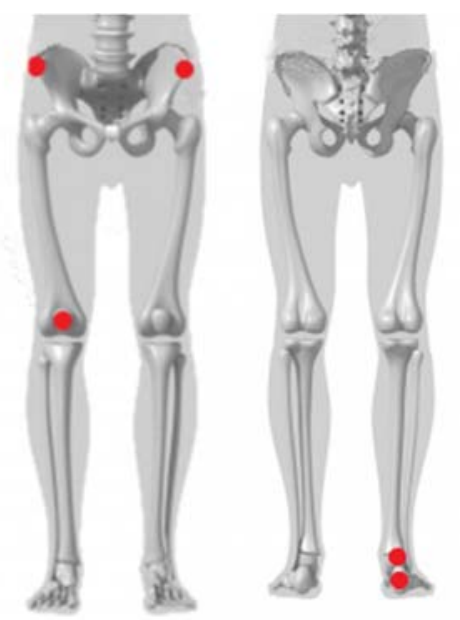

Locations of the five body markers used. 

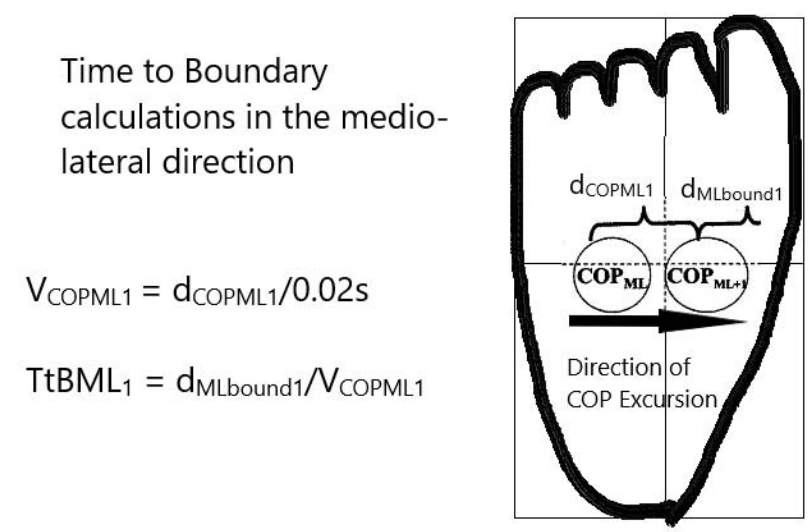

Figure 2

Image adapted from Hertel et al. (2006), Researchers modelled the foot as a rectangle for calculation of time-to-boundary (TtB). This figure shows how TtB was calculated based on center of pressure $(\mathrm{COP})$ excursions in the mediolateral direction.

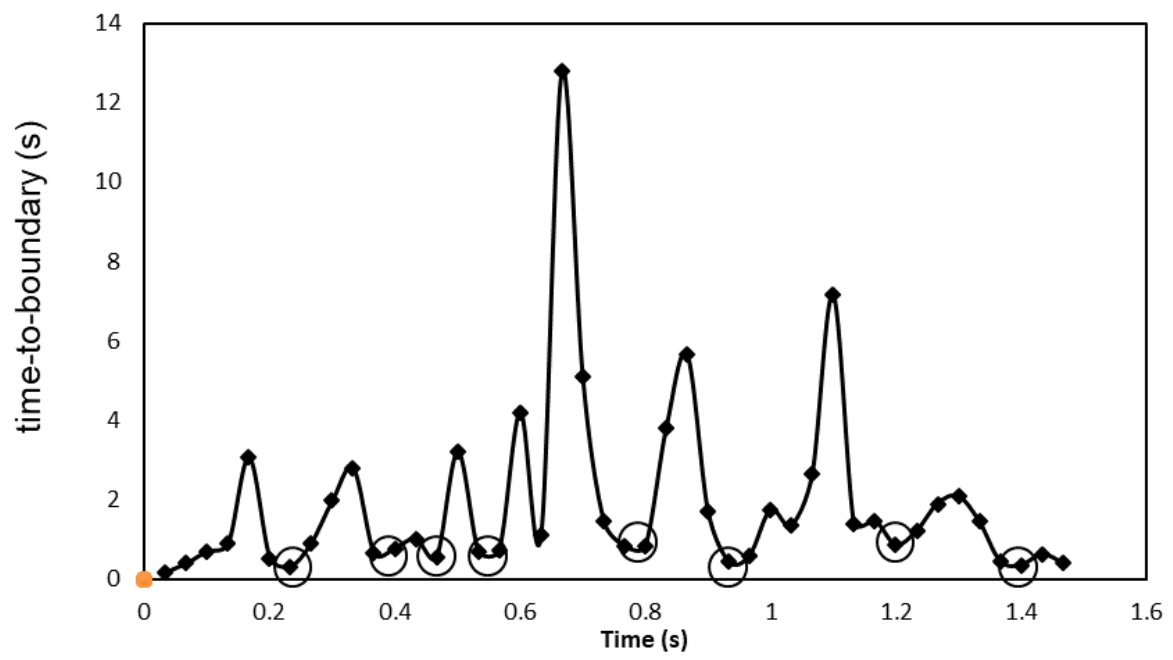

Figure 3

Example of a time-to-boundary data series taken during a single leg lunge over 1.5 s. Minima are indicated in circles; only the lowest values within a valley of TtB data points, as well as singular minimal TtB values qualified as minima. The black line is used purely for visualization of the data. 
Table 1

Descriptive statistics of kinematic data (in degrees) under each condition.

\begin{tabular}{ccccc}
\hline & $\begin{array}{c}\text { Maximum Lateral } \\
\text { Pelvic Tilt }\left(^{\circ}\right)\end{array}$ & $\begin{array}{c}\text { Maximum Knee } \\
\text { Valgus }\left(^{\circ}\right)\end{array}$ & $\begin{array}{c}\text { Maximum Ankle } \\
\text { Eversion }\left(^{\circ}\right)\end{array}$ & $\begin{array}{c}\text { Maximum Ankle } \\
\text { Inversion }\left({ }^{\circ}\right)\end{array}$ \\
\hline Control & 9.1 & 13.84 & 5.74 & 3.5 \\
Light Calf & 9.09 & 14.27 & 5.03 & 4.16 \\
Heavy Calf & 9.09 & 13.54 & 3.51 & 6.2 \\
Heavht Socks & 8.42 & 14.04 & 6.1 & 3.74 \\
Light Leggings & 7.75 & 14.2 & 5.14 & 3.81 \\
Heavy Leggings & 7.99 & 15.02 & 1.9 & 7.31 \\
\hline
\end{tabular}

* significantly greater than control $(p<0.05)$

Table 2

Descriptive statistics of mmTtB (in seconds) under each condition, and standard deviation.

\begin{tabular}{ccc} 
& Mean Minimum TtB (s) & Standard Deviation \\
\hline Control & 0.505 & 0.191 \\
Light Calf & 0.602 & 0.182 \\
Heavy Calf & 0.765 & 0.333 \\
Light Socks & 0.589 & 0.176 \\
Heavy Socks & 0.871 & 0.571 \\
Light Leggings & 0.620 & 0.204 \\
Heavy Leggings & 0.710 & 0.275 \\
\hline
\end{tabular}


Following post-hoc testing, significant differences in mmTtB between Control and Heavy conditions $(-.28 \pm .09 \mathrm{~s} ; p=.007)$, as well as between Light and Heavy $(-.18 \pm .06 \mathrm{~s} ; p=.015)$ were evident. Smaller mmTtB were observed under Control compared to Heavy compression conditions, as well as under Light compared to Heavy compression conditions. No significant differences were found between Control and Light conditions $(p=.802)$ in $\mathrm{mmTtB}$. Standard deviation measures of mmTtB (Table 2) showed that the greatest SD was observed for the Heavy Socks position (SD = .57), while mmTtB SD was lowest for Light Calf conditions $(\mathrm{SD}=.17)$.

\section{Discussion}

The aim of this study was to assess the effects of different compression lengths and tightness on stability and kinematics of the lower body during a forward lunge. The key finding was that there was a significant effect of the garment tightness level on mmTtB. Specifically, mmTtB was significantly greater under Heavy compression compared to Light compression conditions (.18 s); this difference was also the case for mmTtB under Heavy conditions, compared to Control (.28 s). Thus, the initial hypothesis of this research was accepted. However, no significant effects of the compression level or length were evident in any of the lower limb kinematic variables during lunging, therefore rejecting the second hypothesis.

These findings suggest that stability during lunging improved as the compression level increased. This differential effect of tightness when investigating proprioceptive benefits on stability has also been stressed in the concurrent literature (Lee et al., 2016), supporting that tighter garments result in better single-leg stability. Furthermore, this study's results mirror the conclusions reached in recent research by Michael et al. (2014) somewhat; these researchers observed increased stability in a single-leg stance task in participants wearing a well-fitting compression garment, compared to participants wearing a loose compression garment or conventional shorts, stressing that balance was improved due to the enhanced proprioception and a greater cutaneous stimulus, provided by the tighter fit. As such, it seems that there is a convincing argument to be made that 'tighter fitting is better' regarding compression benefits on stability. This is also in line with the data discussed in the relevant garment research focussing on compression garments and stability and joint kinematics (Bernhart and Anderson, 2005; Prymka et al., 1998). Proprioception mainly involves a process of nervous signalling from joint, muscle and deep tissues; these are then projected to the central nervous system, which assures motor control modification (Riemann and Lephart, 2002). Within this paradigm, more intense pressure on the skin may better stimulate the deeper tissues and muscles; as such there is increased information available to the proprioceptor system. It is possible that this increased nervous feedback is what provided benefits to postural control, allowing for better stability of the lead leg during lunging. There was no significant effect observed for garment length on stability suggesting that tighter coverage has a greater influence than more coverage.

Results also revealed no effects of compression garment length or tightness on lower limb kinematics. This was surprising, although not completely unexpected; past research on compression garments and lower extremity biomechanical effects has been mixed in its support of such aids improving movement quality (Pearce et al., 2009). However, an obvious trend was apparent in the data for the lateral pelvic tilt (Table 1); more specifically, smaller angles were calculated as garment length and/or tightness increased. Control averaged a lateral tilt angle of 9.1 degrees (largest measured), while the same angle was 7.4 degrees for lunges in the Heavy Leggings position (smallest measured); however, this is not a statistically significant difference. This would mirror results recently observed in similar ergogenic garment research (Mills et al., 2015). Regardless of that, it can still be argued that the use of such garments can be supported.

It is not possible to compare the absolute values of mmTtB as this has not been used in previous studies examining lunge stability. The absolute values $(0.505-0.871 \mathrm{~s}$ as displayed in Table 2) are less than the values used of greater than $2.00 \mathrm{~s}$ in posture studies (e.g. Hertel et al., 2006; Hertel and Olmsted-Kramer, 2007), but this is to be expected with the performance of a dynamic task. The mmTtB measures have been used for a double leg stance and single leg posture, but this is the first study to use it in a dynamic task. Based 
upon the combination of differences established using this novel method that are not evident in the kinematic data, it is proposed that studies assessing joint stability during balance tests are supplemented with a mmTtB calculation as postural control data related to motor deficiencies may be missed without this center of pressure data. As outlined in the introduction, a dynamic task is more applicable to the sporting world.

All of the data indicate that the heavier condition performed better than the lighter condition. In order to create a heavier compression in this study, two pairs of compression shorts were worn under the "heavy" condition. This creates an argument for athletes wearing two pairs to improve stability when moving in a lunge pattern. While athletes may choose to wear two pairs simultaneously, the manufacturers should consider the compression levels, particularly for short term activity or discrete movement patterns. One anomaly in the data can be seen in the performance of the heavy socks. These data do have a larger mmTtB than both the heavy leggings and the heavy calf. However, due to the large standard deviation associated with this value, it is not possible to see significant differences between the garment types.
As the method using mmTtB continues to develop in the posture literature (e.g. Kim et al., 2019) further consideration should be placed on it in tasks involving dynamic movements. This novel research examining $\mathrm{mmTtB}$ in movement data paves the way for further stability assessments. The observed stability benefits while wearing tight compression garments would prove incredibly useful to research investigating injurious falls and could be applied to neural pathologies and/or injuries that reduce postural control. Longitudinal study of compression clothing use and injury/fall occurrence could also be employed to measure if effects are consistent in real-life athletic situations. Most importantly, the use of tight-fitted compression can be recommended as a method of quickly and easily improving limb stability when executing lunge-like movements, possibly reducing injury and falling risk.

\section{Conclusions}

The results of this study supported that tight compression clothing can improve lower limb stability during a forward lunge. There was no evidence to support any effect of compression garments on kinematics of the lower body. However, as no negative effects through the use of such ergonomic aids have been observed, their usage as a stability aid can be supported.

\section{References}

Blair E, Balluntyne J, Housman S, Chauvel P. A study of a dynamic proximal stability splint in the management of children with cerebral palsy. Dev Med Child Neurol, 1995; 37(6): 544-554

Boyle M. Advances in functional training: training techniques for coaches, personal trainers and athletes. On Target Publications; 2012

Cook G. Movement: Functional Movement Systems. Chichester: Lotus Publishing; 2009

Dascombe BJ, Hoare TK, Sear JA, Reaburn PR, Scanlan AT. The effects of wearing undersized lower-body compression garments on endurance running performance. Int J Sports Physiol Perform, 2011; 6(2): 160-173

Ferber R, Benson B. Changes in multi-segment foot biomechanics with a heat-mouldable semi-custom footorthotic device. J Foot Ankle Res, 2011; 4: 1-8

Floyd R. Manual of Structural Kinesiology. New York: McGraw-Hill; 2012

Hertel J. Functional anatomy, pathomechanics, and pathophysiology of lateral ankle instability. J Athl Train, 2002; 37(4): 364

Hertel J, Olmsted-Kramer LC. Deficits in time-to-boundary measures of postural control with chronic ankle instability. Gait Posture, 2007; 25(1): 33-39

Hertel J, Buckley WE, Denegar CR. Serial testing of postural control after acute lateral ankle sprain. J Athl Train, 2001; 36(4): 363

Hertel J, Olmsted-Kramer LC, Challis JH. Time-to-boundary measures of postural control during single leg quiet standing. J Appl Biomech, 2006; 22(1): 67-73

(c) Editorial Committee of Journal of Human Kinetics 
Inglis JT, Horak FB, Shupert CL, Jones-Rycewicz C. The importance of somatosensory information in triggering and scaling automatic postural responses in humans. Exp Brain Res, 1994; 101(1): 159-164

Kim K-M, Kim J-S, Oh J, Lee SY. Time-to-boundary analysis of postural control following acute lateral ankle sprain. Gait Posture, 2019; 67(1): 151-153

Kritz M, Cronin J, Hume P. Using the body weight forward lunge to screen an athlete's lunge pattern. Strength Cond J, 2009; 31(6): 15-24

Kuster MS, Grob K, Kuster M, Wood GA, Gachter, A. The benefits of wearing a compression sleeve after ACL reconstruction. Med Sci Sports Exerc, 1999; 31: 368-371

Lee H, Hong K, Lee Y. Compression pants with differential pressurisation: Kinetic and kinematical effects on stability. Text Res J, 2016; 87(13): 1554-1564

Mak MK, Ng PL. Mediolateral sway in single-leg stance is the best discriminator of balance performance for Tai-Chi practitioners. Am Arch Rehabil Ther, 2003; 84(5): 683-686

McKeon PO, Hertel J. Systematic review of postural control and lateral ankle instability, part I: can deficits be detected with instrumented testing? J Athl Train, 2008; 43(3): 293-304

Michael JS, Dogramaci SN, Steel KA, Graham KS. What is the effect of compression garments on a balance task in female athletes? Gait Posture, 2014; 39(2): 804-809

Mills C, Knight J, Milligan G. Do ergogenic AIDS alter lower extremity joint alignment during a functional movement lunge prior to and following an exercise bout? J Hum Kinet, 2015; 45(1): 9-17 Paterno MV, Myer GD, Ford KR, Hewett TE. Neuromuscular training improves single-limb stability in young female athletes. J Orthop Sports Phys Ther, 2004; 34(6): 305-316

Paterno MV, Schmitt LC, Ford KR, Rauh MJ, Myer GD, Huang B, Hewett TE. Biomechanical measures during landing and postural stability predict second anterior cruciate ligament injury after anterior cruciate ligament reconstruction and return to sport. Am J Sports Med, 2010; 38(10): 1968-1978

Pearce AJ, Kidgell DJ, Grikepelis LA, Carlson JS. Wearing a sports compression garment on the performance of visuomotor tracking following eccentric exercise: A pilot study. J Sci Med Sport, 2009; 12(4): 500-502

Powers CM. The influence of abnormal hip mechanics on knee injury: a biomechanical perspective. $J$ Orthop Sports Phys Ther, 2010; 40(2): 42-51

Prymka M, Schmidt K, Jerosch J. Proprioception in patients suffering from chondropathia patellae. Int J Sports Med, 1998; 19: 560

Riemann BL, Lephart SM. The Sensorimotor System, Part I: The Physiologic Basis of Functional Joint Stability. J Athl Train, 2002; 37(1): 71-79

Sigel B, Edelstein AL, Savitch L, Hasty JH, Felix Jr. WR. Type of compression for reducing venous stasis: a study of lower extremities during inactive recumbency. Arch Surg, 1975; 110: 171-175

Slobounov SM, Slobounova ES, Newell KM. Virtual time-to-collision and human postural control. J Mot Behav, 1997; 29: 263-281

Van Emmerik REA, Van Wegen EEH. On the functional aspects of variability in postural control. Exerc Sport Sci Rev, 2002; 30: 177-183

Van Wegen EEH, Van Emmerik REA, Riccio GE. Postural orientation: Age-related changes in variability and time-to-boundary. Hum Mov Sci, 2002; 21(1): 61-84

Varela-Sanz A, España J, Carr N, Boullosa DA, Esteve-LanaoJ. Effects of gradual-elastic compression stockings on running economy, kinematics, and performance in runners. J Strength Cond Res, 2011; 25(10): 29022910

\section{Corresponding author:}

\section{Dr Joseph O'Halloran}

School of Sport, Health and Exercise Science, University of Portsmouth; Spinnaker Building

Cambridge Road, Portsmouth, PO1 2ER, United Kingdom

T: +44 (0)2392 84 3087; E-mail: joseph.o'halloran@port.ac.uk 